\title{
Reconstitution de trajectoires de particules solides sur la base d'un modèle de turbulence
}

\author{
Philippe Yvergniaux CEMAGREF, Lyon \\ Jean-Pierre Chollet IMG, Grenoble
}

\section{Introduction}

La plupart des modèles de suspension solide s'expriment en concentrations, la matière solide apparaissant comme un milieu continu régi par des équations de transport et diffusion, les propriétés particulaires (granulométrie, densités) intervenant comme paramètres. Le modèle mathématique présenté ici est au contraire fondé sur une approche particulaire de la suspension: les trajectoires de particules solides sont reconstituées à partir des équations dynamiques régissant leur mouvement ; une difficulté importante est de reconstituer en tout point de la trajectoire de la particule les vitesses instantanées du fluide turbulent environnant. La méthode de "génération lagrangienne de champ turbulent" (adaptée de Desjonquères, 87) prenant en compte les corrélations lagrangiennes de vitesse des particules fluides est présentée ici ; un traitement de la zone de paroi (couche limite) est proposé, permettant de simuler l'effet de «flottement " de particules plus denses que le fluide lié au phénomène des bouffées turbulentes (bursts).

\section{Formulation du mouvement particulaire}

On se base sur l'équation dynamique classique d'une particule solide, due aux travaux de Boussinesq, Basset \& Oseen (BBO, XIX ${ }^{\mathrm{c}}$ ), puis Tchen (1947) et présentée dans sa dernière version, due à Maxey \& Riley (1983) :

$$
\begin{aligned}
& \rho_{p} \cdot \Omega \cdot \mathrm{d} V_{s} / \mathrm{d} t= \\
& -3 / 4 \cdot \Omega \cdot \rho_{f} \cdot C_{D} \cdot\left(V_{s}-V_{f}\right) \cdot\left\|V_{s}-V_{f}\right\| / \phi \\
& \text { (traînée de Stokes ) } \\
& -\frac{3 \cdot \rho_{f} \cdot \Omega \cdot \sqrt{v} \cdot C_{H}}{2 \phi \sqrt{\pi}} \cdot \int_{-\infty}^{t} \frac{\mathrm{d}\left(V_{s}-V_{f}\right) / \mathrm{d} t}{\begin{array}{c}
\sqrt{\left(t-t^{\prime}\right)} \\
(\text { terme de Basset })
\end{array}} \\
& +\left(\rho_{p}-\rho_{f}\right) \cdot \Omega \cdot g \\
& -\rho_{f} \cdot \Omega \cdot C_{A} \cdot \mathrm{d}\left(V_{s}-V_{f}\right) / \mathrm{d} t \quad \text { (masse ajoutée) } \\
& +\rho_{f} \cdot \Omega \cdot D V_{f} / D t \quad \text { (pression) }
\end{aligned}
$$

où : $\Omega=$ volume de la particule, $\rho_{p}=$ masse volumique de la particule, $\rho_{f}=$ masse volumique du fluide, $V_{s}=$ vitesse de la particule solide, $V_{f}=$ vitesse du fluide environnant, $\phi=$ diamètre de la particule, $v=$ viscosité cinématique du fluide $\left(=10^{-6}\right.$ pour l'eau), $C_{D}=$ coefficient de traînée $=24 / R_{e p} \cdot\left(1+0,15 \cdot R_{e p}^{0.687}\right)$, valable pour $R_{e p}<200$ (Clift, 1978).

$$
\begin{aligned}
& C_{H}=2,88+3,12 /\left(A_{c}^{1 / 2}+1\right)^{3}, \\
& C_{A}=1,05-0,066 /\left(A_{c}^{2}+0,12\right),
\end{aligned}
$$

valables pour $R_{e p}<60$ (Odar et Hamilton, 1964), avec

$$
A_{c}=\frac{\left\|V_{s}-V_{f}\right\|}{\phi \cdot \frac{\left\|\mathrm{d}\left(V_{s}-V_{f}\right)\right\|}{\mathrm{d} t}}
$$

$R_{e p}=\frac{\left|V_{s}-V_{f}\right| \cdot \phi}{v}$ est le nombre de Reynolds particulaire

$$
\mathrm{d} / \mathrm{d} t=\delta / \delta t+V_{s i} \cdot \delta / \delta x_{i}
$$

(dérivée suivant le mouvement de la particule solide)

$$
D / D t=\delta / \delta t+V_{f i} \cdot \delta / \delta x_{i}
$$

(suivant le fluide).

La validité de l'équation (1) nécessite que le fluide soit homogène au voisinage de la particule solide ; dans un écoulement turbulent, cela revient à considérer que $\phi$ est au plus de l'ordre de grandeur de l'échelle de Kolmogorov, $\eta=\left(v^{3} / \varepsilon\right)^{1 / 4}$, où $\varepsilon$ est le taux de dissipation turbulente. Nous nous placerons dans ce cadre pour les développements suivants.

L'écoulement test choisi est un écoulement à surface libre, de $1 \mathrm{~m}$ de profondeur, de vitesse moyenne $1 \mathrm{~m} / \mathrm{s}$, sur fond lisse. Dans ces conditions, $\eta$ varie de $50 \mu \mathrm{m}$ à $1 \mathrm{~mm}$ de la paroi à $3000 \mu \mathrm{m}$ au cœur de l'écoulement) ; les particules solides suivies sont de taille comprise entre 50 et $1000 \mu \mathrm{m}$. 


\section{Particle trajectories modelling based on a standard model of turbulence}

Most of the suspension modellings work on concentrations, the solid phase being considered as a continuous state governed by convection and diffusion equations; on the contrary, the numerical model presented here is based on a particle approach of suspension: the trajectory of a solid particle is built timestep after timestep, using the dynamic equation of a solid particle in a fluid flow; an important difficulty is to rebuild, at each timestep, the fluid velocity field surrounding the solid particle. A method of "lagrangian generation of local turbulent field " (adapted from Desjonquères 87) is presented here, which simulates fluid trajectories in conserving lagrangian velocity correlations, and then calculates the solid particle trajectories. We suggest a method for simulating the behaviour of relatively heavy solid particles in the boundary layer, taking the "slipping effect " (mainly due to the bursting process) into account.

\section{Mouvement d'une particule fluide}

La résolution de l'équation (1) nécessite de connaître les composantes de la vitesse instantanée de la particule fluide coïncidant avec la particule solide en $(X, t)$. La simulation directe de l'écoulement turbulent (très coûteuse en temps de calcul) ne permet pas de simuler à la fois les grandes et les petites échelles de la turbulence ; la démarche adoptée ici est différente :

1) Un code classique calcule les valeurs moyennes du champ de vitesse: $U_{m}, V_{m}, \bar{u}^{2}, \bar{v}^{2}, \bar{w}^{2}, \overline{u v}, \overline{u w}$, $\overline{v w}$ (où $U(X, t)=U_{m}(X, t)+u(X, t)$ suivant la décomposition de Taylor); on utilise ici le code VEST $(k-\varepsilon, 2 D)$ de Rodi (Stamou-Adams, 1985), qui donne $U_{m}, V_{m}, k, \varepsilon$ plus une équation algébrique pour le calcul du tenseur de Reynolds (Rodi, 81). Les valeurs pour la couche limite (non calculable par un code $k-\varepsilon$ qui se limite à $y^{+}>30$ ) sont tirées de lois standard (Kay et Nedderman, 74, pour $U_{m}$, Laufer, 54, pour $\bar{u}^{2}, \bar{v}^{2}$, $\bar{w}^{2}, \overline{u v}$, Rodi, 81 pour $k$ et $\varepsilon$ ). Nous utiliserons :



où $u^{*}=$ vitesse de frottement $\approx C_{\mu}^{1 / 4} \cdot k^{1 / 2}$

$C_{\mu}=0,09, K=0,42 \quad$ (constante de von Karman).
2) Les valeurs instantanées $u, v, w(X, t)$ des fluctuations de vitesse sont obtenues par tirage de variables aléatoires $\Gamma_{u}, \Gamma_{v}, \Gamma_{w}$; on impose par ailleurs le respect des corrélations lagrangiennes de la particule fluide, prises sous la forme de Frenkiel (1948):

$$
\begin{aligned}
\overline{u_{1}(t) \cdot u_{1}(t+\mathrm{d} t)}=\sqrt{\overline{u_{1}^{2}}(t)} \cdot \sqrt{\overline{u_{1}^{2}(t+\mathrm{d} t)}} \times \\
\times \exp \left(\mathrm{d} t / 2 \tau_{1}\right)
\end{aligned}
$$

où $\tau_{1}$ est le temps de corrélation lagrangienne du fluide, pris sous la forme: $\tau_{1}=C_{t} \cdot \overline{u^{2}} / \varepsilon\left(C_{t} \approx 0,2-0,6\right.$, voir Picart 84); pour $\mathrm{d} t=0$ on retrouve les corrélations en 1 point.

Le respect de ces corrélations est assuré par la factorisation de Cholesky de la matrice $A$ des corrélations lagrangiennes $\quad\left(A_{i j}=\overline{u\left(t^{\prime}\right) \cdot u(t)} / \sqrt{\overline{u^{2}}\left(t^{\prime}\right)} \cdot \sqrt{\overline{u^{2}}(t)}\right)$, qui amène à exprimer la vitesse instantanée d'une particule fluide à l'étape $n$ de sa trajectoire sous la forme :

$$
U_{n}=\bar{U}_{n}+\sum_{k=1}^{n}\left(B_{n k} \cdot \Gamma_{k}\right) .
$$

Les $\Gamma_{k}$ sont des variables aléatoires (prises gaussiennes en général). On se limite numériquement aux corrélations jusqu'à $\left(t^{\prime}-t\right)=10 \cdot \tau_{1}$.

Des exemples de trajectoires de particules fluides ainsi calculées sont donnés dans la figure 1 (pour un taux de turbulence $u / U_{m}$ de $15 \%$, soit $k \approx 0,2$ pour $U_{m}=1$ ).

$\left(^{*}\right)$ L'adjonction d'un facteur $\cos \left(\mathrm{d} t / 2 \tau_{1}\right)$, contestable sur le plan théorique (Färber 85, Pope 88), peut améliorer la qualité des résultats (Hinze 75, Picart et al. 86).

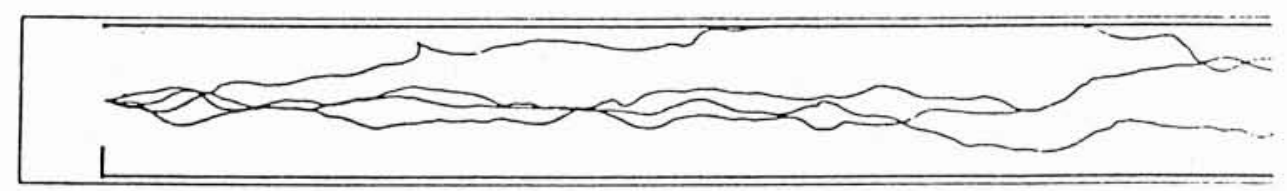

1. Trajectoires de particules fluides $\left(u / U_{m}=15 \%\right)$. 


\section{Mouvement d'une particule solide}

Le mouvement de la particule solide est obtenu par intégration à chaque pas de temps de l'équation (1). En pratique, on suit une même particule fluide $F$ tant que la distance de celle-ci à la particule solide $S$ donnée reste inférieure à $L e$ (échelle intégrale eulérienne $\left.\approx C \cdot \sqrt{u^{2}} \cdot \tau_{1}\right)$, la vitesse de la particule fluide $F S(t)$ coïncidant avec $S$ à l'instant $t$ étant tirée au sort en respectant les corrélations eulériennes $\overline{U F S . U F}$ de type $\exp (-D / 2 L e)$; au-delà, on reprend une nouvelle particule fluide $F$ (sans mémoire, ce qui permet de simuler les effets de croissement de trajectoires).

(1) est discrétisée suivant un schéma "pseudo-implicite $"$ :

$$
\begin{aligned}
\left(U_{s}(t+\mathrm{d} t)-\right. & \left.U_{s}(t)\right) / \mathrm{d} t=A \cdot\left(U_{s}(t+\mathrm{d} t)+\right. \\
& \left.+U_{s}(t)\right) / 2+(B(t+\mathrm{d} t)+B(t)) / 2 .
\end{aligned}
$$

La condition de stabilité étant $A \cdot \mathrm{d} t<0,2$, soit $\mathrm{d} t / \tau_{p}<$ 0,2 où $\tau_{p}$ est le temps caractéristique de la particule :

$$
\tau_{p}=\phi^{2} \cdot(\delta+1 / 2) / 18
$$

où $\delta=\rho_{p} / \rho_{f} \quad\left(\right.$ pour $\left.\phi=10^{-4}, \tau_{p}=10^{-3}\right)$.

En pratique on utilise les pas de temps suivants :

$\mathrm{d} t_{f}=\tau_{1} / 5$ à $\tau_{1} / 2$ pour la particule fluide

$\mathrm{d} t_{s}=\tau_{p} / 10$ pour la particule solide (en général, $\left.\mathrm{d} t_{s} \ll \mathrm{d} t_{f}\right)$.

On néglige également le terme de Basset la plupart du temps (dès que $\tau_{p}<\tau_{s}$, d'après Gouesbet, 81 ).

L'inconvénient de la méthode proposée vient de ce que la particule fluide suivie $F$ (pour laquelle on respecte les corrélations lagrangiennes) n'est pas la particule $F S$ coïncidant avec la particule solide $S$; en particulier entre deux pas de temps $i$ et $i+1$, on respecte

$\overline{U F(i) . U F S(i)}, \overline{U F(i) \cdot U F(i+1)}$,

$$
\overline{U F(i+1) \cdot U F \bar{S}(i+1)} \text {, }
$$

mais pas $\overline{U F S(i) \cdot \operatorname{UFS}(i+1)}$; cet inconvénient ne peut être évité que si l'on pouvait connaitre à tout instant le champ de vitesses dans tout l'espace; des essais de champs analytiques chaotiques permettant de simuler le champ turbulent au même degré de précision que la méthode présentée ici ont été entrepris qui pourraient donner à terme des résultats.

\section{Comportement des particules solides près du fond}

5.1. La modélisation de la trajectoire solide près du fond présente deux niveaux de difficulté :

- d'une part, les forces rentrant en jeu dans l'équation dynamique sont encore mal connues : ceci est en particulier vrai pour les particules relativement grosses $(\phi \gg \eta)$ qui interagissent avec plusieurs particules fluides et modifient notablement la turbulence en leur voisinage (ainsi la mise en évidence récente de "negative lifts " ou forces dirigées vers la paroi, voir Willetts \& Murray, 81, notamment) ; dans notre cas on supposera (à l'instar de Sumer, 85) que pour les particules suffisamment petites qui nous intéressent, l'équation (1) reste valable ; notons cependant que près de la couche tampon le taux de turbulence est relativement important et l'échelle de Kolmogorov réduite d'autant (dans le cas test, $k$ $10^{-2}$ et $\eta=50 \mu \mathrm{m}$ à $y^{+}=30$, soit $\left.y=1 \mathrm{~mm}\right)$;

- d'autre part, l'écoulement fluide lui-même est très complexe et encore assez mal connu et quantifié; néanmoins on a identifié et étudié depuis une quinzaine d'années le phénomène des "bursts ", ou bouffées turbulentes, qui apparaît comme le principal élément de la structure turbulente de la couche limite et joue un rôle important dans le démarrage et le maintien en suspension des particules solides (Offen-Kline, 75, Cantwell, 81, Sumer-Deigaard, 81); on peut le décrire brièvement ainsi : à intervalles irréguliers (dans le temps et l'espace) se produit une suite d'événements bien déterminés dont le cycle forme un «burst "; il comprend :

- pénétration de fluide à forte vitesse de la zone tampon $\left(5<y^{+}<70\right.$ en gros) vers la paroi, provoquant la naissance d'un «rouleau» ou «éclair» à faible vitesse ;

- balayage puis remontée vers $y^{+} \sim 100$ de cet éclair ; - éclatement de l'éclair après quelques oscillations dans la couche logarithmique $\left(y^{+} \sim 100\right)$.

Lors du passage des bursts près de la paroi, la tension de frottement peut atteindre 60 fois sa valeur moyenne, ce qui peut provoquer l'éjection de particules solides relativement lourdes du fond.

5.2. La possibilité pour une particule solide de "flotter " au voisinage du fond (en fait de se déplacer par bonds sans toucher le fond, voir graph. 2, d'après SumerDeigaard, 81)) a été expérimentalement reliée à la valeur du paramètre $\theta=W_{c} / K u^{*}$ par rapport à 1 , où $W_{c}=(\delta-1) \cdot g \cdot \phi^{2} / 18$, vitesse limite de chute de la particule solide ; Sumer (1985), se référant aux travaux de Batchelor (65), explique ce comportement du fait que près de la paroi on a $\sqrt{v^{2}} \approx u^{*}$, et donc que la vitesse



2. Trajectoires expérimentales de particules solides près du fond (d'après Sumer-Deigaard, 81). 


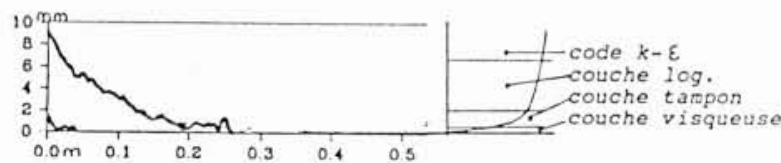

$3 a$



3. Trajectoires modélisées de particules solides près du fond: $3 a$ : distribution gaussienne de vitesses verticales; $3 b$ : distribution en loi exponentielle.

verticale du fluide est $V_{f}=\alpha \cdot u^{*}$, ce qui intégré dans l'équation (1) donne :

$$
\begin{aligned}
\mathrm{d} V_{s} / \mathrm{d} t=-\left(V_{s}-V_{f}\right) \cdot 18 \cdot v / \phi^{2} \cdot(\delta+1 / 2)- \\
-(\delta-1) g /(\delta+1 / 2),
\end{aligned}
$$

qui admet comme solution $V_{s}=0$ si $W_{c} / V_{f}=1$.

Cependant la vitesse verticale du fluide fluctue et est de moyenne nulle; les essais faits sur notre code de calcul montrent que si l'on prend par exemple une distribution de vitesse symétrique (gaussienne par ex.) les particules ne " rebondissent " pas sur la couche limite et atteignent le fond très rapidement (graph. $3 a$ );

Par contre, si l'on introduit une fluctuation de vitesse verticale dissymétrique (moment d'ordre 3 ou " skewness ", négatif) on arrive à obtenir ce phénomène de «flottement " de particules solides de densité supérieure à 1 ; le graphique $3 b$ illustre le cas suivant :

- champ fluide :

$$
U_{m}=1 \mathrm{~m} / \mathrm{s}, \quad u^{*}=0,035 \mathrm{~m} / \mathrm{s}
$$

- particule solide :

$$
\begin{gathered}
\delta=1,22, \quad \phi=500 \mathrm{~m}, \quad W_{c}=0,030 \mathrm{~m} / \mathrm{s}, \quad \theta=2 \\
y(t=0)=9.10^{-4} \mathrm{~m}\left(y^{+}=33\right)
\end{gathered}
$$

- loi de distribution de $V_{f}$ exponentielle :

$$
\begin{gathered}
F\left(x>x^{\bullet}\right)=1-\exp \left(\left(x-x^{\bullet}\right) / a\right) \\
\left(m(V>0)=0,6 \sqrt{v^{2}}, \quad m(V<0)=\sqrt{v^{2}}\right) .
\end{gathered}
$$

Ce comportement dissymétrique rend par ailleurs bien compte du phénomène même des bursts : en un point donné de la plage $0<y^{+}<70$ où les bouffées turbulentes se développent, les vitesses verticales négatives du fluide sont d'intensité plus forte que les vitesses positives (éclairs descendants à forte vitesse et montants à faible vitesse), et (par conservation de la masse) de fréquence plus faible; l'inertie de la particule solide face à ces sollicitations dissymétriques du fluide environnant fait que l'on obtient une force résultante moyenne positive, qui, si elle équilibre la force de gravité, -assure le maintien au-dessus du fond.

\section{Conclusion}

Nous avons exposé une méthode qui permet de reconstituer les trajectoires de particules solides dans un écoulement turbulent, et de simuler notamment qualitativement l'action des bursts sur celles-ci ; toutefois il reste encore, pour caler ces méthodes quantitativement, à les confronter à des résultats de mesures, malheureusement très rares pour la zone de la couche limite : mesures de distribution de fluctuations de vitesses dans la zone tampon, trajectographies pour des particules de petite taille.

\section{Références}

DESJONQUĖRES Ph., 1987: Modélisation lagrangienne du comportement de particules discrètes en écoulement turbulent. Thèse, Rouen.

FÄRBER K., 1985 : Investigations of particle motions in turbulent flow. Euromech 192, pp. 33-36.

Hinze J. O., 1959: Turbulence. McGraw-Hill, New-York.

MAXEY M. R. and RILEY J. J., 1983 : Equation of the motion of a small rigid sphere in a non-uniform flow. Phys. fluids, vol. 26, pp. 883-889.

Picart A., Berlemont A. and Gouesbet G., 1986 : Modelling and predicting turbulence fields and the dispersion of discrete particles transported by turbulent flows, Int. J. Multiph. Flows, n 2, pp. 237-261.

RoDI W., 1981: Turbulence models and their applications in hydraulics. I.A.H.R. ed. 1984.

SUMER B. M., 1984 : Lift forces on moving particles near boundaries. J.H.E., vol. 110-9, pp. 1272-1278.

SUMER B. M., 1985 : Recent developments on the mechanics of sediment suspension. Euromech 192, pp. 3-14.

Sumer B. M. and DeigaARd R., 1981: Particle motion near the bottom in turbulent flow in an open channel. J.F.M., vol. 109, pp. 311-337.

TCHEN C. M., 1947: Equation of the motion for a particle suspended in an homogeneous field. Ph. D. Univ. Delft. 